\title{
THE INFLUENCE OF A SMALL CARDINAL ON THE PRODUCT OF A LINDELÖF SPACE AND THE IRRATIONALS
}

\author{
L. BRIAN LAWRENCE
}

(Communicated by R. Daniel Mauldin)

\begin{abstract}
It is unknown whether there is in ZFC a Lindelöf space whose product with the irrationals is nonnormal. We give some necessary conditions based on the minimum cardinality of $a \leq^{*}$ unbounded family in ${ }^{\omega} \omega$.
\end{abstract}

\section{INTRODUCTION}

History. In 1963 [9], E. A. Michael gave the first ZFC example of a normal space and a metric space with a nonnormal product, where the term "space" is an abbreviation for "Hausdorff regular topological space." (In 1955 [12], M. E. Rudin used a Souslin line to construct a Dowker space-a normal space whose product with the closed unit interval is nonnormal; the existence of a Souslin line was shown to be independent of ZFC in the late 1960s. Rudin constructed a real Dowker space in 1971 [13].) Let $\mathbb{P}, \mathbb{Q}$, and $\mathbb{R}$ denote respectively the irrationals, rationals, and reals with their usual topologies; and let $\mathbb{M}$ denote the Michael line which is the refinement of $\mathbb{R}$ obtained by isolating each irrational point. In Michael's example, $\mathbb{M}$ is the normal space and $\mathbb{P}$ is the metric space; $\mathbb{M}$ is moreover hereditarily paracompact.

A topological space $X$ is concentrated on a subset $A \subseteq X$ if for every open set $U \supseteq A, X \backslash U$ is countable. The Continuum Hypothesis implies the existence of an uncountable subset $X \subseteq \mathbb{R}$ with $X$ concentrated on $\mathbb{Q}$ (A.S. Besicovitch [2], 1934; in fact, $\underline{b}=\omega_{1}$ is all his proof requires-see Propositions 2 and 3 below). Michael obtained this result independently and pointed out that as a subspace of $\mathbb{M}, X$ is Lindelöf whereas $X \times \mathbb{P}$ is nonnormal ([10], 1971; first mentioned in a footnote of [9]).

Received by the editors May 5, 1989 and, in revised form, November 2, 1989; a part of this paper was presented at the Northeast Topology Conference held at Wesleyan University in June 1988. Sponsors included the National Science Foundation and the New York Academy of Sciences. 1980 Mathematics Subject Classification (1985 Revision). Primary 54B10; Secondary 54D20, 54E50.

Key words and phrases. Product, irrationals, separable completely metrizable space, Lindelöf space, normal, Michael line, concentrated space, Continuum Hypothesis, Martin's axiom.

The author gratefully acknowledges support received from the Howard University Faculty Research Support Grant Program. 
Also in the 1963 paper, Michael gave a ZFC example of a Lindelöf space $\mathbb{M}^{\prime}$ and a separable metric space $S$ with $\mathbb{M}^{\prime} \times S$ nonnormal. Let $A$ be a Bernstein set in $\mathbb{R}$; that is, choose $A$ so that each of $A$ and $\mathbb{R} \backslash A$ intersects every uncountable closed set. (A set of this type was first constructed by F. Bernstein in 1908 using transfinite recursion and the Cantor-Bendixson theorem: each of the $\underline{c}$-many closed sets in $\mathbb{R}$ is either countable or has cardinality $\underline{c}$, where $\underline{c}=2^{\omega}$; see [11, pp. 23-24.]) Note that $\mathbb{R}$ is concentrated on $A$. Let $\mathbb{M}^{\prime}$ be the refinement of $\mathbb{R}$ obtained by isolating each point of $\mathbb{R} \backslash A$, and let $S$ be $\mathbb{R} \backslash A$ with the subspace topology (note that $S$ is not complete).

Is there a real Lindelöf space whose product with $\mathbb{P}$ is nonnormal? This is equivalent to the existence in ZFC of a Lindelöf space and a separable completely metrizable space with a nonnormal product (see Proposition 1 below).

Michael spaces. Define a Michael space to be a Lindelöf space whose product with $\mathbb{P}$ is nonnormal. Is there a Michael space in ZFC? In particular, can we build a real Michael space by the method of isolating points in a separable metric space? Define a concentrated Michael space to be a Michael space that is concentrated on a closed subset $A$ where $A \times \mathbb{P}$ is normal. This concept captures the construction of isolating points mentioned above, in which case $A$ is the derived set (i.e., the closed subset of nonisolated points) of the refinement; since $A$ is a (separable) metrizable subspace of the refinement, its product with $\mathbb{P}$ is normal.

In his handbook article ("The Integers and Topology" in [7, pp. 111-167, and in particular, pp. 150-153]), E. K. van Douwen noted some necessary conditions for the existence of a real Michael space which are the point of departure for the current paper. We first establish that regardless of special settheoretic axioms, a Michael space cannot be constructed by isolating points in an initial space that is completely metrizable (Theorem 1). We then show that the existence of a concentrated Michael space is independent of ZFC (Theorem 2 ). Finally, we give a lower bound for the cardinality and weight of an arbitrary Michael space (Theorem 3). (The weight of a space $X$, denoted by $w(X)$, is the minimum cardinality of a base for the topology of $X$.) As in the case of the results in the van Douwen article, the cardinal number $\underline{b}$ (defined below) is at the heart of the matter in Theorems 2 and 3.

\section{Propositions}

Remark. The propositions below are now more or less standard. We sketch the proofs for the convenience of the reader.

Proposition 1. Suppose $X$ and $Y$ are spaces where $Y$ is separable, completely metrizable, and not $\sigma$-compact. Then $X \times Y$ is normal iff $X \times \mathbb{P}$ is normal.

Proof. Sufficiency follows from three standard theorems: Normality is preserved under a closed continuous map (see [4, p. 69]); every separable completely metrizable space is the image under a perfect map of a closed subspace of $\mathbb{P}$ (a map is perfect if it is closed and continuous, and each point-inverse set 
is compact); and, a Cartesian product of perfect mappings is perfect [4, p. 237]. (Since $\mathbb{P}$ is homeomorphic to ${ }^{\omega} \mathbb{P}[4$, p. 348$]$, and every separable completely metrizable space can be embedded as a closed subspace in ${ }^{\omega} \mathbb{R}[4$, p. 342], we can prove the second theorem by using $\omega$-many copies of the Cantor ternary function [7, pp. 203-204] to define a perfect map from a closed subspace of $\mathbb{P}$ onto $\mathbb{R}$, and in turn, from a closed subspace of ${ }^{\omega} \mathbb{P}$ onto ${ }^{\omega} \mathbb{R}$.)

Necessity. $Y$ has a closed subspace homeomorphic to $\mathbb{P}$ (W. Hurewicz; see van Douwen's proof in [7, pp. 141-142]).

Notation. Recall that $\mathbb{P}$ can be identified with ${ }^{\omega} \omega=\{f \mid f: \omega \rightarrow \omega\}$ with the product topology: for each $\xi \in{ }^{<\omega} \omega=\{\eta \mid \eta:[0, n] \rightarrow \omega$ for some $n \in \omega\}$, $\left\{f \in{ }^{\omega} \omega: \xi \subseteq f\right\}$ is a basic open set (see [7, p. 204]). For $f, g \in{ }^{\omega} \omega$, define $f \leq g$ if for each $n \in \omega, f(n) \leq g(n)$; and define $f \leq^{*} g$ if there exists $m \in \omega$ such that for each $n \geq m, f(n) \leq g(n)$. For each $g \in{ }^{\omega} \omega$, let $C(g)=\left\{f \in{ }^{\omega} \omega: f \leq g\right\}$, and let $C^{*}(g)=\left\{f \in{ }^{\omega} \omega: f \leq^{*} g\right\}$.

Compact sets. (1) For each $g \in{ }^{\omega} \omega, C(g)$ is compact and $C^{*}(g)$ is $\sigma$ compact.

(2) For each $T \subseteq{ }^{\omega} \omega$, if $T$ is compact (resp., $\sigma$-compact), then there exists $g \in{ }^{\omega} \omega$ such that $T \subseteq C(g)$ (resp., $T \subseteq C^{*}(g)$ ) (Note that the compactness relationship implies the analogue for $\sigma$-compactness, since for any sequence $g: \omega \rightarrow{ }^{\omega} \omega, \bigcup_{k \in \omega} C\left(g_{k}\right) \subseteq C^{*}(h)$ where $\left.h(n)=\sum_{k \leq n} g_{k}(n)\right)$.

(3) For all $f, g \in{ }^{\omega} \omega, C(f) \subseteq C(g)$ (resp., $\left.C^{*}(f) \subseteq C^{*}(g)\right)$ iff $f \leq g$ (resp. $f \leq^{*} g$ ).

Cardinals. With respect to $\leq^{*}$, let $\underline{b}$ be the minimum cardinality of an unbounded family and let $\underline{d}$ be the minimum cardinality of a dominant (cofinal) family. Then in ZFC we have:

(1) $\omega_{1} \leq \underline{b} \leq \underline{d} \leq \underline{c}$;

(2) $\underline{b}$ is regular because there is a $\leq^{*}$ well-ordered unbounded family of order type $\underline{b}$. (To prove this, let $f: \underline{b} \rightarrow{ }^{\omega} \omega$ with Range $(f)$ unbounded, and then define $g: \underline{b} \rightarrow{ }^{\omega} \omega$ recursively so that for all $\alpha, \beta \in \underline{b}$ with $\alpha<\beta$, $\left.f(\alpha) \leq^{*} g(\alpha) \leq^{*} g(\beta)\right)$;

(3) $\underline{b}=\underline{d}$ iff there is a $\leq^{*}$ well-ordered dominant family (often called a scale). (To prove sufficiency, note that if $g: \gamma \rightarrow{ }^{\omega} \omega$ is an order-isomorphism onto a dominant subcollection, then $\underline{d} \leq$ cofinality of $\gamma \leq \underline{b}$; and to prove necessity, let $f: \underline{b} \rightarrow{ }^{\omega} \omega$ with $\operatorname{Range}(f)$ dominant, and then define $g$ as in the proof that $\underline{b}$ is regular.)

The Continuum Hypothesis implies $\omega_{1}=\underline{b}=\underline{d}=\underline{c}$, while under Martin's axiom, $\underline{b}=\underline{d}=\underline{c}$ (see [6, p. 87, Example 8]). Since Martin's axiom is consistent with the negation of the Continuum Hypothesis [6, pp. 278-281], we have that the combinatorial statement $\underline{b}=\omega_{1}$ is independent of ZFC.

Consistency results. In [3], D. K. Burke and S. Davis constructed a Michael space using an $\omega_{1}$-scale (equivalently, $\underline{d}=\omega_{1}$ ); while in [7], van Douwen 
obtained a Michael space under $\underline{b}=\omega_{1}$. The hypotheses $\underline{c}=\omega_{1}, \underline{d}=\omega_{1}$, and $\underline{b}=\omega_{1}$ are successively weaker statements (see [5]).

Proposition 2. Suppose $X$ is an uncountable subset of $\mathbb{P}$. Then the following are equivalent:

(1) every uncountable subset of $X$ is $\leq^{*}$ unbounded in ${ }^{\omega} \omega$;

(2) $X$ has countable intersection with every compact subset of $\mathbb{P}$;

(3) $X \cup \mathbb{Q}$ is concentrated on $\mathbb{Q}$;

(4) $X \cup \mathbb{Q}$ with the subspace topology of $\mathbb{M}$ is Lindelöf.

Proposition 3. There is an uncountable subset of $\mathbb{P}$ satisfying each condition of Proposition 2 iff $\underline{b}=\omega_{1}$.

Proof of Proposition 2. Each of (1) and (2) is equivalent to $X$ having countable intersection with $C^{*}(g)$ for every $g \in{ }^{\omega} \omega$.

For (2) implies (3), note that a closed set in $\mathbb{R}$ which is contained in $\mathbb{R} \backslash \mathbb{Q}$ is a $\sigma$-compact subset of $\mathbb{P}$ and therefore is contained in $C^{*}(g)$ for some $g \in{ }^{\omega} \omega$. The converse follows from the fact that the complement in $\mathbb{R}$ of a compact subset of $\mathbb{P}$ is an open set in $\mathbb{R}$ containing $\mathbb{Q}$.

The equivalence of (3) and (4) is immediate from the definitions.

Proof of Proposition 3. Suppose $X$ is an uncountable subset of $\mathbb{P}$ satisfying the first condition of Proposition 2. Let $Y \subseteq X$ with $|Y|=\omega_{1}$. By hypothesis, $Y$ is unbounded so $\underline{b}=\omega_{1}$. For the converse, let $X$ be a $\leq^{*}$ well-ordered unbounded family in ${ }^{\omega} \omega$ of type $\omega_{1}$.

\section{THEOREMS}

Lemma 1. (Rudin and M. Starbird, [14], 1975). The product of a Lindelöf space and a separable metric space is normal iff it is Lindelöf.

Lemma 2 (Characterization). Suppose $S$ is a separable metric space and $X$ is a Lindelof space that is concentrated on a closed subset $A \subseteq X$ where $A \times S$ is normal. Then $X \times S$ is normal iff for every uncountable subset $B \subseteq X \backslash A$ and $1-1$ function $F: B \rightarrow S, \operatorname{cl}(\operatorname{Graph}(F)) \cap(A \times S) \neq \varnothing$.

Proof. Sufficiency of the contrapositive. We will show that $\operatorname{cl}(\operatorname{Graph}(F))$ and $A \times S$ cannot be separated by disjoint open sets. Let $U$ be an open set in the product with $A \times S \subseteq U$. Let $D$ be a dense countable subset of $S$. For each $d \in D$, let $Y_{d}=\{x \in X:\langle x, d\rangle \notin U\}$. By the concentration of $X$ on $A$, each $Y_{d}$ is countable, and since $B$ is uncountable, we can choose $x_{0} \in B \backslash \bigcup_{d \in D} Y_{d}$. Then $\left\{x_{0}\right\} \times D \subseteq U$, so $\left\langle x_{0}, F\left(x_{0}\right)\right\rangle \in \operatorname{cl}(U)$.

Necessity of the contrapositive. By Lemma 1 in application to $A$ and $S$, we have that $A \times S$ is Lindelöf. So if every open cover of $A \times S$ also covers each point of all but countably many vertical sections (i.e., the point-inverse sets under projection to $X$ ), then $X \times S$ is Lindelöf.

Suppose $X \times S$ is nonnormal. Then by the preceding paragraph, we can choose an open set $U \supseteq A \times S$ such that the complement of $U$ intersects uncountably many vertical sections. For each $s \in S$, again let $Y_{s}=\{x \in$ $X:\langle x, s\rangle \notin U\}$, and let $Z=\bigcup_{s \in S} Y_{s}$. Then each $Y_{s}$ is countable while 
$Z$ is uncountable, so we can define $\sigma: \omega_{1} \rightarrow Z$ and $\tau: \omega_{1} \rightarrow S$ by transfinite recursion so that for each $\alpha \in \omega_{1}, \sigma(\alpha) \in Y_{\tau(\alpha)} \backslash \bigcup_{\beta<\alpha} Y_{\tau(\beta)}$. Let $B=\operatorname{Range}(\sigma)$ and let $F=\left\{\langle\sigma(\alpha), \tau(\alpha)\rangle: \alpha \in \omega_{1}\right\}$.

Notation. Suppose $S$ is a separable metric space and $A \subseteq S$ where $S$ is concentrated on $A$. Define $\mathbb{L}(S, A)$ to be the Lindelöf refinement of $S$ obtained by isolating each point of $S \backslash A$.

Corollary 1. Suppose $S$ is a separable metric space and $A \subseteq S$, where $S$ is concentrated on $A$ and $S \backslash A$ is uncountable. Then $\mathbb{L}(S, A) \times(S \backslash A)$ is nonnormal (where $S \backslash A$ has the subspace topology).

Proof. Use Lemma 2 with $F$ the identity function on $S \backslash A$. (This choice of closed sets that cannot be separated is the prototypical construction discovered by Michael.)

Remark. In Lemma 3, and consequently in Theorem 1 and Corollary 2 also, $\mathbb{P}$ can be replaced by any separable completely metrizable space.

Lemma 3. Suppose $Y$ is a separable completely metrizable space, and $A \subseteq X \subseteq$ $Y$, where $X$ is concentrated on $A$ and $\mathbb{L}(X, A) \times \mathbb{P}$ is nonnormal. Then there is a Cantor set $K \subseteq Y$ such that $K$ is disjoint from $A$ while contained in the $Y$ closure of $A$.

Proof. Choose $F$ according to Lemma 2. Let $G$ be the closure of $\operatorname{Graph}(F)$ in the topology of $Y \times \mathbb{P}$. Note that if $U$ is an open set in $\mathbb{L}(X, A) \times \mathbb{P}$ containing $A \times \mathbb{P}$, then there is a set $V \supseteq A \times \mathbb{P}$ which is open in $Y \times \mathbb{P}$ and satisfies $U \supseteq V \cap(X \times \mathbb{P})$; so if $U \cap \operatorname{Graph}(F)=\varnothing$, then $V \cap \operatorname{Graph}(F)=\varnothing$. Thus $G$ is disjoint from $A \times \mathbb{P}$.

For the remainder of the proof we will work entirely in the topology of $Y \times \mathbb{P}$. We need the following theorem: Every uncountable subset $T$ of a separable metric space contains a condensation point (i.e., there exists $t \in T$ such that for each open set $U$ containing $t,|U \cap T| \geq \omega_{1}$ ). (For a proof, assume otherwise and use the Lindelöf property on the subspace to obtain a countable open cover of $T$ where each open set contains only countably many points of $T$.)

By the theorem just quoted, we can choose a sequence $\left\langle U_{n}: n \in \omega\right\rangle$ of open sets in the product $Y \times \mathbb{P}$ such that $U_{0}$ is a basic ("rectangular") open set, and for each $n>0$ :

(1) $\operatorname{cl}\left(U_{n}\right) \subseteq U_{n-1}$;

(2) each component of $U_{n-1}$ is a basic open set with diameter $<\frac{1}{n}$, and contains at least two, but no more than a finite number of, components of $U_{n}$

(3) the projections to $Y$ of the components of $U_{n}$ are pairwise disjoint; and

(4) each component of $U_{n}$ has uncountable intersection with $\operatorname{Graph}(F)$. 
Let $H=\bigcap_{n \in \omega} U_{n}$. Then by the completeness of $Y \times \mathbb{P}, H$ is a Cantor set which is contained in $G$ and intersects uncountably many vertical sections. Let $K$ be the projection of $H$ to $Y$. Then $K \cap A=\varnothing$, while $K$ is contained in the $Y$ closure of $A$ since $X$ is concentrated on $A$ and each neighborhood of each point of $K$ has uncountable intersection with $X$.

Theorem 1. Suppose $Y$ is a separable completely metrizable space concentrated on a subset $A$. Then $\mathbb{L}(Y, A) \times \mathbb{P}$ is normal (and therefore Lindelöf by Lemma 1) in $Z F C$.

Proof. The concentration of $Y$ on $A$ implies that $A$ intersects every Cantor set contained in $Y$; so by the contrapositive of Lemma $3, \mathbb{L}(Y, A) \times \mathbb{P}$ is normal.

Corollary 2. Suppose $A$ is a Bernstein set in $\mathbb{R}$. Then $\mathbb{L}(\mathbb{R}, A) \times \mathbb{P}$ is Lindelöf in $Z F C$.

Remark. Suppose $A \subseteq \mathbb{R}$ is Bernstein, and give both $A$ and $\mathbb{R} \backslash A$ the subspace topology. By Corollaries 1 and $2, \mathbb{L}(R, A) \times \mathbb{P}$ is Lindelöf whereas $\mathbb{L}(\mathbb{R}, A) \times$ $\mathbb{R} \backslash A$ is nonnormal. However, completeness in $\mathbb{P}$, or its failure in $\mathbb{R} \backslash A$, is not the only relevant property. The status of $\mathbb{L}(\mathbb{R}, A) \times A$ depends upon the choice of $A$. For each $n$ with $n \in \omega$ or $n=\omega$, there is a Bernstein set $A$ such that for each $m<n, \mathbb{L}(\mathbb{R}, A) \times{ }^{m} A$ is Lindelöf, whereas $\mathbb{L}(\mathbb{R}, A) \times{ }^{n} A$ is nonnormal (see [8] for the proof).

Theorem 2. There is a concentrated Michael space iff $\underline{b}=\omega_{1}$.

Proof.

Sufficiency. Suppose $\underline{b}=\omega_{1}$. Use Propositions 2 and 3 to choose an uncountable Lindelöf subspace $X$ of $\mathbb{M}$ with $\mathbb{Q} \subseteq X$. Then $X \times \mathbb{P}$ is nonnormal by Lemma 2 using the Michael construction: $S=\mathbb{P}, A=\mathbb{Q}, B=X \backslash A$, and $F$ is the identity function on $B$.

Necessity. Suppose $X$ is a Lindelöf space that is concentrated on a closed subset $A \subseteq X$ where $A \times \mathbb{P}$ is normal. Suppose further that $X \times \mathbb{P}$ is nonnormal. Choose $F$ according to Lemma 2. Claim. Range $(F)$ has countable intersection with every compact subset of $\mathbb{P}$. If the claim holds, then $\underline{b}=\omega_{1}$ by the propositions.

Assume the claim is false and let $T$ be a compact subset of $\mathbb{P}$ and let $G$ be a restriction of $F$ such that $|\operatorname{Graph}(G)|=\omega_{1}$ and $\operatorname{Range}(G) \subseteq T$. For each $a \in A$, let $W(a)$ be a finite basic open cover of $\{a\} \times T$, where each element is disjoint from $\operatorname{cl}(\operatorname{Graph}(G))$. Let $V=\left\{\bigcap_{U \in W(a)} \operatorname{Proj}_{X}(U): a \in A\right\}$ (where $\operatorname{Proj}_{X}$ is the projection function to $X$ ). Then $V$ is an open cover of $A$ with Domain $(G) \cap \bigcup V=\varnothing$, which contradicts the concentration of $X$ on $A$.

Corollary 3. A real Michael space cannot be concentrated on a subset whose product with $\mathbb{P}$ is Lindelöf whether or not the subset is closed.

Proof. Suppose $X$ is a Lindelöf space concentrated on a subset $A \subseteq X$ where $A \times \mathbb{P}$ is Lindelöf. Let $Y$ be the refinement of $X$ obtained by isolating each 
point of $X \backslash A$. By Theorem 2 and Lemma 1, if $\underline{b}>\omega_{1}$, then $Y \times \mathbb{P}$ is Lindelöf; and since $X \times \mathbb{P}$ has a coarser topology, it too must be Lindelöf.

Corollary 4. The derived set of a real Michael space is itself a Michael space. Proof. A Lindelöf space is concentrated on its derived set, so Corollary 3 applies.

Lemma 4. Suppose $X$ is a Lindelöf space. Then for each $g \in{ }^{\omega} \omega, X \times C^{*}(g)$ is a Lindelöf subspace of $X \times \mathbb{P}$.

Proof. The result follows from two facts: (1) the product of a Lindelöf space and a compact space is Lindelöf and (2) $C^{*}(g)$ is $\sigma$-compact.

Notation. Let $\underline{\ell}$ be the minimum of $\underline{b}$ and $\operatorname{lub}\left\{\omega_{n}: n \in \omega\right\}$. (We are not using the customary symbol for the above least upper bound to avoid confusion with our use of ${ }^{\omega} \omega$ for the function space.)

Theorem 3. Suppose $X$ is a Michael space. Then $|X| \geq \underline{\ell}$ and $w(X) \geq \underline{\ell}$.

Proof. Claim. $L(X \times \mathbb{P}) \geq \ell$ (where $L(X \times \mathbb{P})$ is the Lindelöf degree of $X \times \mathbb{P}$ which is defined to be the minimum of $\{\kappa$ : every open cover of $X \times \mathbb{P}$ has a subcover of cardinality $\leq \kappa\})$. Before proving the claim, first note that the theorem is an immediate consequence since $L(X \times \mathbb{P}) \leq \min (|X|, w(X))$, which in turn follows from $w(X \times \mathbb{P})=w(X)$ and the fact that each vertical section of $X \times \mathbb{P}$ is a Lindelöf subspace.

Proof of the claim. Let $\Lambda$ be an open cover of $X \times \mathbb{P}$ of minimum cardinality which does not have a countable subcover, and let $\underline{\lambda}=|\Lambda|$. Note that $L(X \times \mathbb{P})$ $\geq \underline{\lambda}$. We will show that either $\underline{\lambda}$ has countable cofinality or $\underline{\lambda} \geq \underline{b}$. Thus $\underline{\lambda} \geq \underline{\ell}$.

Assume $\underline{\lambda}$ does not have countable cofinality, and let $\theta: \underline{\lambda} \rightarrow \Lambda$ be a $1-1$ correspondence. By transfinite recursion we now define strictly increasing sequences $\sigma: \underline{b} \rightarrow{ }^{\omega} \omega$ and $\tau: \underline{b} \rightarrow \underline{\lambda}$ (where ${ }^{\omega} \omega$ is partially ordered by $\leq^{*}$ ) such that for each $\alpha \in \underline{b}, X \times C^{*}\left(\sigma_{\alpha}\right)$ is not entirely covered by $\left\{\theta_{\beta}: \beta<\right.$ lub Range $(\tau \mid \alpha)\}$, but is covered by $\left\{\theta_{\beta}: \beta<\tau_{\alpha}\right\}$.

Suppose $\gamma \in \underline{b}$ and $\sigma \mid \gamma$ and $\tau \mid \gamma$ have been defined so as to satisfy the above condition. For the extension of $\sigma$ we consider two cases. Let $\varepsilon=$ lub Range $(\tau \mid \gamma)$. If $\gamma$ has countable cofinality, then $\varepsilon<\underline{\lambda}$ by our starting assumption on $\underline{\lambda}$, so the existence of an appropriate choice for $\sigma_{\gamma}$ follows from $\underline{b} \geq \omega_{1}$ and the minimality in the choice of $\Lambda$. Assume that $\gamma$ does not have countable cofinality. Let $\sigma_{\gamma}$ be a $\leq^{*}$ upper bound for $\sigma \mid \gamma$. If $X \times C^{*}\left(\sigma_{\gamma}\right)$ is covered by $\left\{\theta_{\beta}: \beta<\varepsilon\right\}$, then by Lemma 4 there is a countable subcover, which by our assumption on $\gamma$ implies the existence of $\delta<\gamma$ such that $X \times C^{*}\left(\sigma_{\gamma}\right)$ is covered by $\left\{\theta_{\beta}: \beta<\tau_{\delta}\right\}$; this contradicts our hypothesis since $C^{*}\left(\sigma_{\delta+1}\right) \subseteq C^{*}\left(\sigma_{\gamma}\right)$. For the extension of $\tau$, again use Lemma 4 and the uncountable cofinality of $\underline{\lambda}$.

Corollary 5. Suppose $X$ is a first countable Michael space. Then under Martin's axiom together with $\underline{c}=\omega_{n}$ for some $n \in \omega,|X|=w(X)=\underline{c}$. 
Proof. By Archangel'skii's theorem (see [7, p. 19]), $\underline{c}$ is an upper bound for the cardinality and weight of a first countable Lindelöf space; and since Martin's axiom implies $\underline{b}=\underline{c}$, we have by Theorem 3 that $\underline{c}$ is also a lower bound.

Remark. Recently K. Alster showed in [1] that Martin's axiom implies the existence of a Michael space. Since $\underline{b}=\underline{c}$ under Martin's axiom and $\underline{c} \neq \omega_{1}$ is consistent with Martin's axiom, Alster's example cannot be concentrated. Thus the existence of a Michael space that is not concentrated is consistent with ZFC.

Problems. (1) Extend the independence result of Theorem 2 to a larger class of spaces. In particular, is the existence of a Michael space obtained as a refinement of a separable metric space independent of ZFC?

(2) Can Theorem 3 be improved by replacing $\underline{\ell}$ with $\underline{b}$ ?

(3) In both the Michael and Alster examples, one type of point and open set yields the Lindelöf property, while a second type guarantees nonnormality in the product with $\mathbb{P}$. Is there a homogeneous Michael space in ZFC?

\section{ACKNOWLEDGMENT}

The author thanks the referees for a number of very helpful suggestions.

\section{REFERENCES}

1. K. Alster, On the product of a Lindelöf space and the space of irrationals under Martin's Axiom, Proc. Amer. Math. Soc. 110 (1990), 543-547.

2. A. S. Besicovitch, Concentrated and rarified sets of points, Acta Math. 62 (1934), 289-300.

3. D. K. Burke and S. Davis, Subsets of ${ }^{\omega} \omega$ and generalized metric spaces, Pacific J. Math. 110 (1984), 273-281.

4. R. Engelking, General topology, Polish Scientific Publishers, 1977.

5. S. H. Hechler, On the existence of certain cofinal subsets of ${ }^{\omega} \omega$, Proc. Sympos. Pure Math. Vol. 10-2, Amer. Math. Soc., Providence, RI, 1974, pp. 155-174.

6. K. Kunen, Set theory: an introduction to independence proofs, North-Holland, 1980.

7. K. Kunen and J. E. Vaughan, eds., Handbook of set-theoretic topology, North-Holland, 1984.

8. L. B. Lawrence, Lindelöf spaces concentrated on Bernstein subsets of the real line (to appear).

9. E. A. Michael, The product of a normal space and a metric space need not be normal, Bull. Amer. Math. Soc. 69 (1963), 375-376.

10. , Paracompactness and the Lindelöf property in finite and countable cartesian products, Comp. Math. 23 (1971), 199-214.

11. J. C. Oxtoby, Measure and category, Springer-Verlag, 1970.

12. M. E. Rudin, Countable paracompactness and Souslin's problem, Canad. J. Math. 7 (1955), 543-547.

13. _ A normal space $X$ for which $X \times I$ is not normal, Fund. Math. 73 (1971), 179-186.

14. M. E. Rudin and M. Starbird, Products with a metric factor, Topology Appl. 5 (1975), 235-248.

Department of Mathematics, Howard University, Washington, DC 20059 22030

Current address: Department of Mathematics, George Mason University, Fairfax, Virginia 